\title{
Análise Sobre Redes Ópticas com Heurísticas de Roteamento, Modulação e Alocação de Espectro para Tráfego Incremental
}

\author{
Leonardo A. J. Mesquita ${ }^{1}$, Karcius D. R. Assis ${ }^{1}$ \\ ${ }^{1}$ Departamento de Engenharia Elétrica - Universidade Federal da Bahia (UFBA) \\ Salvador 40210-630, BA, Brazil \\ ajm.leonardo@hotmail.com, karcius.assis@ufba.br
}

\begin{abstract}
The advances in optical data transmission technology have allowed the great expansion of the internet and bandwidth demanding services. The emergence of orthogonal frequency-division multiplexing (OFDM) has opened the possibility of increasing the network's spectral efficiency through the resolution of the routing and spectrum allocation (RSA) problem. In this work, we implemented two RSA heuristics, which accounts for different available modulation formats (RMSA). Simulations were made for various networks, applying the algorithms, under incremental traffic conditions. The results have shown that the load balancing allocation heuristic (BMLM) displayed a smaller initial blocking rate, when only one modulation format was available, but showed greater long-term blocking rate, relative to the shortest path allocation heuristic (SPMLM). When more modulation formats were available, the BMLM presented worse performance in most of the cases, but managed better results in a few networks.
\end{abstract}

Resumo. Os avanços na tecnologia de transmissão óptica de dados permitiram o grande crescimento da Internet e de serviços exigentes em largura de banda. O surgimento das redes baseadas em multiplexação por divisão de frequências ortogonais (OFDM) abriram a possibilidade de se aumentar a eficiência espectral de uma rede através da resolução do problema de roteamento e alocação de espectro (RSA). Neste trabalho, foram implementadas duas heurísticas de RSA que consideram diferentes formatos de modulação disponíveis (RMSA). Simulações foram feitas para diversas redes, usando as heurísticas, em condições de tráfego incremental. Os resultados mostraram que a heurística de alocação por balanceamento de carga (BMLM) tendeu a apresentar menor taxa de bloqueio nos períodos iniciais, quando havia apenas um formato de modulação disponível, mas apresentou maior taxa de bloqueio, a longo prazo, relativa à heurística por alocação de caminho mais curto (SPMLM). Quando mais formatos de modulação estavam disponíveis, o BMLM apresentou pior desempenho na maioria dos casos, mas conseguiu melhores resultados em algumas redes.

\section{Introdução}

Os últimos anos têm conhecido um grande aumento em novos serviços na internet, desde novas plataformas de entretenimento e redes sociais a transações financeiras e blockchain. Pesquisas realizadas pela Cisco mostraram que a taxa de dados transmitida apresentará um crescimento composto de $26 \%$ ao ano entre 2017 e 2022 [Cisco 2017]. 
O crescimento da banda disponível permitiu o advento de novos modelos de negócios na Internet como TV em alta definição, streaming de vídeo, computação em nuvem, mídia social, blockchain, localização geográfica em tempo real e outros serviços comuns que exigem diferentes níveis de qualidade de serviço (Quality of Service - QoS) e largura de banda.

Redes ópticas elásticas (Spectrum-sliced Elastic Optical Networks - SLICE), baseadas em multiplexação por divisão de frequências ortogonais (Orthogonal Frequency Division Multiplexing - OFDM), são uma proposta promissora para lidar com a diversidade de tráfego, pois possuem maior granularidade das suas portadoras unitárias de frequência, conhecidas como slots de frequência (SF), o que dá a essas redes uma maior eficiência espectral [Jinno et al. 2009, Fontinele et al. 2018, Agrawal 2017]. Além disso, redes baseadas em OFDM fornecem um maior nível de flexibilidade, reconfigurabilidade e facilidade de ampliação, que já são características exigidas dos provedores de Internet (Internet Service Provider - ISP) atualmente e serão ainda mais importantes no futuro [Zhang et al. 2013].

Junto com a maior granularidade das redes SLICE também existe uma maior complexidade durante o roteamento de tráfego. Para rotear as demandas de tráfego, o algoritmo deve considerar não só os caminhos a serem percorridos, mas também quais serão os SF atribuídos a cada uma das conexões. Esse desafio é conhecido como o problema de roteamento e alocação de espectro (Routing and Spectrum Assignment - RSA) [Pioro and Medhi 2004], que é explicado em maior detalhe na Seção 2.

Quando diferentes formatos de modulação podem ser usados para diferentes conexões, a depender da distância percorrida entre os dados, ao RSA é adicionado a complexidade da escolha dos formatos de modulação, que podem reduzir a banda usada, mas que geralmente estão limitados a uma distância de transmissão, o que resulta no problema chamado de roteamento, modulação e alocação de espectro (Routing Modulation and Spectrum Assignment - RMSA) [Abkenar and Rahbar 2017].

A resolução do RMSA, para otimizar uma determinada métrica da rede como, por exemplo, a carga média utilizada ou a taxa de bloqueio (TdB) de conexões, é o objetivo de vários trabalhos publicados neste campo e que podem ser de grande ajuda para administradores que buscam melhorar a performance e a utilização dos recursos de suas redes.

Um dos maiores objetivos, com relação à manutenção de redes, é aumentar a longevidade dos recursos e adiar a instalação de novos cabos e dispositivos como multiplexadores ópticos e transponders, que costumam consumir bastante tempo e dinheiro. Ter que instalar fibras precocemente, pode gerar um desperdício de recursos, já que a capacidade de transmissão das fibras costuma dobrar a cada dois anos [Agrawal 2013]. Por isso, o planejamento apropriado em um longo horizonte de tempo, é um fator estratégico e de crítica importância para empresas do setor de comunicação.

Para avaliar um cenário de exaustão de recursos, a melhor opção é a simulação de tráfego incremental. Assume-se que durante alguns períodos discretos de tempo, a rede precisa servir uma nova leva de conexões. Cada um desses períodos de novas demandas será chamado de período de tráfego incremental, onde a rede deverá atender a um conjunto de novas demandas de tráfego, em que cada uma será alocada e mantida indefinidamente. 
Neste trabalho, propomos um algoritmo que usa heurísticas para resolver o RMSA e fazer a alocação de SFs nos enlaces da rede, dada uma matriz de tráfego. Esse algoritmo pode usar a mesma rede ou substrato físico, e novas matrizes de tráfego, referentes a novas demandas, para simular o tráfego incremental. O objetivo é analisar como se comporta a taxa de bloqueio de diversas redes, para consecutivos incrementos de tráfego; e observar como ocorre o esgotamento dos SFs nos enlaces de acordo com o funcionamento das heurísticas.

O estudo foi feito comparando duas heurísticas, cujo funcionamento é análogo às heurísticas que costumam aparecer na literatura [Wang et al. 2011], adaptadas para usar diferentes formatos de modulação (FdM). A primeira heurística se chama Shortest Path with Minimum Load Modulation (SPMLM) e a segunda foi nomeada de Balanced Minimum Load Modulation (BMLM). Simulações foram realizadas com essas heurísticas para duas conjecturas de FdMs disponíveis. Os resultados são apresentados na Seção 5 e levantam considerações interessantes sobre a aplicabilidade das heurísticas, a depender de diferentes características das redes.

\section{Roteamento, Modulação e Alocação de Espectro}

Redes ópticas baseadas em OFDM possuem a vantagem de serem mais flexíveis e possuírem uma maior granularidade de seu espectro de frequência, em comparação com as redes baseadas em multiplexação por divisão de comprimento de onda (Wavelength Division Multiplexing - WDM) que têm uma divisão fixa da banda, geralmente de 50 Ghz, intercaladas por bandas de guarda, como no padrão da ITU-T [ITU-T 2002]. Em redes OFDM, as divisões de banda são mais modulares, geralmente $12.5 \mathrm{Ghz}$ [Christodoulopoulos et al. 2011] e, para uma mesma conexão, as portadoras podem ser atribuídas adjacentes umas às outras, sem precisar de portadoras intermediárias entre os SFs como banda de guarda. Também, por possuir SFs mais granulares, são melhores em servir conexões de diferentes larguras de banda. A combinação desses fatores permite uma maior eficiência espectral nas redes elásticas.

Em redes SLICE, os dados são roteados sem sofrer nenhum tipo de conversão óptica-elétrica-óptica. Logo, não é possível fazer conversão de espectro, o que significa que quando uma conexão é roteada de um nó fonte a um nó destino, os mesmos SFs devem ser usados ao longo do conjunto de enlaces de fibra dessa rota. Essa condição é conhecida como a restrição de continuidade. Outra restrição presente em redes SLICE é que, para uma mesma conexão, todos os SFs usados devem ser adjacentes uns aos outros. Essa é a restrição de contiguidade de slots.

Quando uma rede SLICE precisa rotear uma conexão entre dois nós, além de considerar a rota, ela deve considerar as restrições de continuidade e contiguidade. Isso define o problema conhecido como roteamento e alocação de espectro (RSA).

A tecnologia OFDM também permite a escolha do número de símbolos de transmissão, por bit (Formato de modulação) [Christodoulopoulos et al. 2011], que pode ser realizado com o mesmo transponder. Como a eficiência espectral de uma conexão depende diretamente da distância de trasmissão, o formato de modulação (FdM) usado deve ser adaptável à distância de roteamento. Com essa consideração, existe uma complexidade adicional, pois o roteamento também deve levar em conta qual FdM é mais eficiente para percorrer toda a rota. 
A Tabela 1 mostra os formatos de modulação usados para as simulações na Seção 5. Os valores da tabela estão condizentes com [Oliveira et al. 2017] e [Jaya et al. 2016].

Tabela 1. Formatos de Modulação

\begin{tabular}{lcccc}
\hline $\begin{array}{c}\text { Formato } \\
\text { de } \\
\text { Modulação }\end{array}$ & Taxa de bits & $\begin{array}{c}\text { Distância } \\
\text { Máxima } \\
(\mathrm{km})\end{array}$ & $\begin{array}{c}\text { Eficiência } \\
\text { Espectral } \\
(\mathrm{bps} / \mathrm{hz})\end{array}$ & $\begin{array}{c}\text { Banda } \\
\text { por } \\
\text { Portadora } \\
(\mathrm{Ghz})\end{array}$ \\
\hline 16QAM & $1 / 4$ & 500 & 4 & 12,5 \\
\hline 8QAM & $2 / 4$ & 1000 & 3 & 12,5 \\
\hline QPSK & $3 / 4$ & 2000 & 2 & 12,5 \\
\hline BPSK & 1 & 4000 & 1 & 12,5 \\
\hline
\end{tabular}

É importante notar que a banda consumida por uma demanda irá depender do caminho pelo qual ela será roteada. Suponha que cada portadora ou SF ocupe uma banda de $B \mathrm{GHz}$ e uma conexão de $t$ Gbits por segundo seja roteada por um formato de modulação que possua uma eficiência espectral igual a $\eta$ Gbps por GHz. A Fórmula 1 dá o número de SFs necessários $(\tau)$, para atender essa conexão, caso esse formato de modulação seja usado. Como o quociente do lado direito da fórmula pode ser uma fração, ele é aproximado para o número inteiro logo acima dele, pois $\tau$ deve ser um número inteiro. A função teto que faz essa aproximação é representada por $\lceil *\rceil$.

$$
\tau=\left\lceil\frac{t}{\eta \times B}\right\rceil
$$

É possível notar, pela Fórmula (1), que o número de slots será menor, quando a eficiência do formato de modulação for maior. No entanto, maior eficiência implica menor alcance para a conexão, como pode ser visto na Tabela 1. Quando as restrições do RSA são combinadas a essas novas considerações, o problema se chama roteamento, modulação e alocação de espectro (RMSA). A Figura 1 ilustra um exemplo de resolução do RMSA em uma rede em anel com 4 nós.
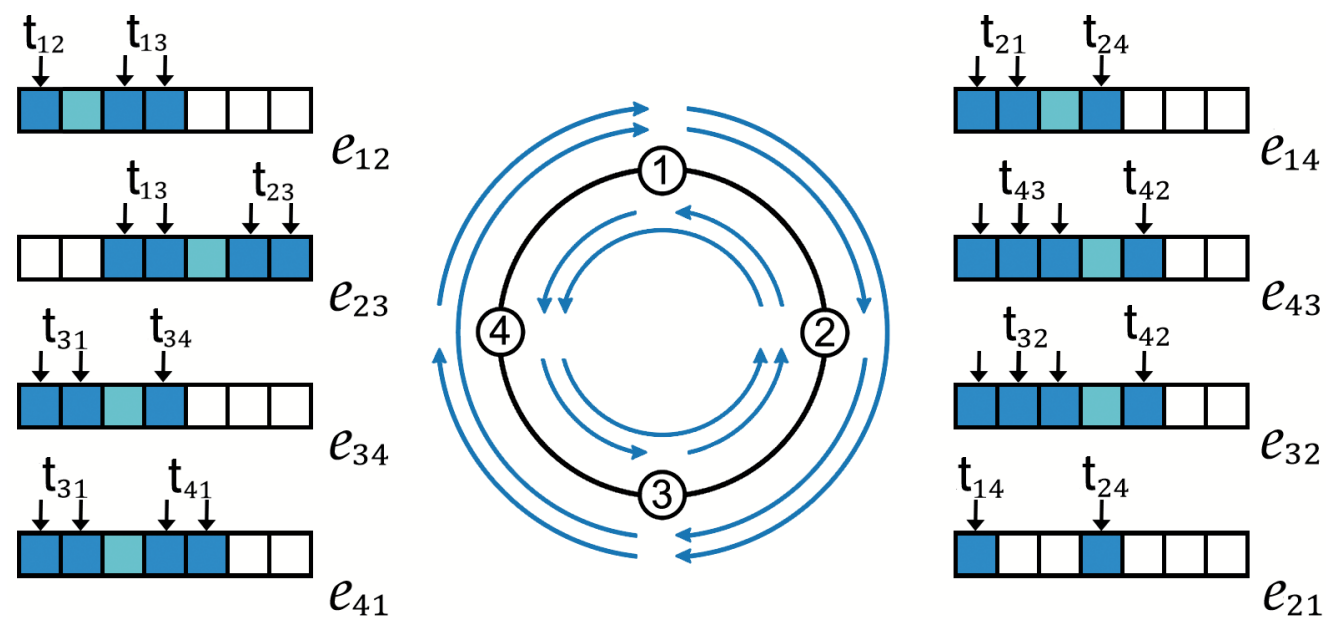

Figura 1. Exemplo de resolução de RMSA em uma rede anel com 4 nós. 
Na Figura 1, a rede e os nós estão coloridos de preto. Os nós estão enumerados de $1 \mathrm{a} 4$. As setas direcionais de cor azulada simbolizam a rota que um determinado tráfego percorre, partindo do nó fonte para o nó destino. O conjunto de SFs de cada um dos oito enlaces $e_{i j}$ estão à esquerda e à direita da rede. As caixas preenchidas são os SFs alocados para uma demanda de tráfego $t_{i j}$ indicada logo acima. As caixas preenchidas sem indicação são referentes à banda de guarda.

Suponha que uma matriz de tráfego $T=\left\{t_{i j} \in \mathbb{N}^{4 \times 4}\right\}$, com as demandas de tráfego em Gbps, deva ser alocada na rede da Figura 1. Cada elemento $t_{i j}$ dessa matriz, sendo um número natural, representa a demanda do nó $i$, para o nó $j$.

$$
T=\left[\begin{array}{cccc}
0 & 50 & 50 & 25 \\
70 & 0 & 65 & 30 \\
60 & 120 & 0 & 45 \\
100 & 35 & 150 & 0
\end{array}\right]
$$

Vamos supor que o algoritmo que fará o RMSA, na rede anel, seja uma heurística de roteamento que sempre escolha o caminho mais curto para rotear a demanda atual. Imagine que, para este algoritmo, os formatos de modulação 8QAM e 16QAM estejam passíveis de serem usados e que cada enlace tenha uma distância de $500 \mathrm{~km}$. Suponha também que a política de alocação de SFs escolha sempre os primeiros slots livres que possam alocar aquela carga (política conhecida como First Fit - FF) e que o número de slots, de banda de guarda, entre as alocações seja igual a 1.

A heurística terá que usar uma ordem para alocar essas demandas. Neste caso, serão escolhidos primeiro os elementos, da matriz, da esquerda para direita e de cima para baixo (sentido de leitura).

O primeiro elemento válido para se alocar seria a demanda de $50 \mathrm{Gbps}$, do nó 1 para o nó 2. O caminho mais curto seria usando apenas o enlace $e_{12}$. Como a distância total é igual a $500 \mathrm{~km}$, pode-se usar 16QAM como FdM. Logo, usando a Fórmula 1, um slot será alocado no enlace $e_{12}$. Em seguida, a próxima demanda é do nó 1 para o nó 3, também de 50 Gbps. O número de SFs consumidos será 2. Existem dois caminhos mais curtos, então, para esta heurística, qualquer um dos dois pode ser usado. Neste caso, o caminho do nó 1 para o nó 2 para o nó 3 será escolhido. A distância total é de $1000 \mathrm{~km}$, então não poderá ser usado 16QAM como FdM. Assim, usando 8QAM, 2 SFs contíguos devem ser alocados de forma contínua nos enlaces $e_{12}$ e $e_{23}$. Note, na Figura 1, que a demanda $t_{13}$ teve de ser alocada depois da banda de guarda no enlace $e_{12}$. Por isso, no enlace $e_{23}$, os dois primeiros slots ficaram vazios, já que, pela restrição da continuidade, as mesmas portadoras devem ser alocadas para todos os enlaces de uma mesma conexão.

Seguindo o mesmo padrão para os outros elementos da matriz de tráfego, ao final, a resolução do RMSA, feita pela heurística será como na Figura 1. Note que no enlace $e_{23}$ somente o primeiro slot pode ser preenchido, pois o segundo slot deve ser usado como banda de guarda. Além disso, no enlace $e_{21}$, o segundo e terceiro slots não poderão ser utilizados em momento algum, pois não há como inserir nenhuma demanda junto com os dois slots de banda de guarda necessários. A esse efeito, em que surgem SFs dispersos de difícil alocação, se dá o nome de fragmentação de espectro [Silva et al. 2018] e é uma consequência inevitável das restrições de continuidade e contiguidade. 


\section{Tráfego Incremental}

Uma rede pode ser dimensionada a partir de uma demanda estimada previamente ou por meio de modelos estatísticos, para prever qual será a demanda exigida da rede no futuro e projetá-la com o intuito de minimizar a probabilidade de bloqueio a partir desses dados. Esse planejamento usa modelos para tráfego tido como estático. As simulações para tráfego estático são mais simples, no entanto, esta metodologia não pode fornecer informação sobre como a probabilidade de bloqueio da rede irá se comportar ao longo do tempo.

A análise da rede sob condições de tráfego dinâmico, ou incremental, são propostas mais realistas e que fornecem relatórios mais completos sobre a qualidade de serviço e o consumo de recursos da rede. Nas condições de tráfego dinâmico, novas conexões podem surgir ou desaparecer com o tempo e a rede deve alocar ou desalocar os SFs de acordo. No tráfego incremental, novas conexões surgem com o tempo e devem ser alocadas, sem desaparecer, simulando assim as condições de maior exaustão da rede. O que fornece, aos operadores, uma perspectiva do pior cenário de escassez de recursos.

[Straub et al. 2006] mostrou que o planejamento de redes pela abordagem de tráfego incremental é superior do ponto de vista custo-benefício à abordagem estática, em arquiteturas WDM. [Velasco et al. 2016] considerou o planejamento da expansão da capacidade da rede em regime multiperíodo, para previsões de demanda de tráfego médio crescente com ajuda de alguns modelos matemáticos. [Soumplis et al. 2017] apresentou um algoritmo para fazer o planejamento de redes em condições de tráfego incremental e mostrou até 36\% de melhoras em gastos. [Iyer and Singh 2018] propôs formulações de ILP para minimizar o custo de operação em redes multicamadas, considerando tráfego incremental. Esses trabalhos mostram resultados promissores para o estudo de redes elásticas em regime multiperíodo.

Neste trabalho, o tráfego incremental foi modelado como uma série temporal, onde uma matriz $T=\left\{t_{i j} \in \mathbb{N}^{v \times v}\right\}$ é um conjunto de demandas de tráfego relativas a um período de alocação ( $v$ é o número de nós da rede). Desta forma, outras matrizes similares podem ser geradas, relativas a outros períodos de tráfego incremental e, assim, teremos as taxas de bloqueio da rede para cada período de incremento de tráfego. A partir deste modelo, é possível observar como os recursos da rede são esgotados para os múltiplos períodos. As notações e o algoritmo são melhores descritos na Seção 4.

\section{Algoritmo de RMSA Incremental}

O problema do RMSA pode ser resolvido de forma ótima, a minizar uma determinada métrica requerida, com o uso de formulações de programação linear inteira (Integer Linear Programming - ILP) como a proposta em [Jia et al. 2015]. No entanto, um dos pontos negativos de se usar tais formulações é que o tempo de execução cresce exponencialmente em função do número de nós e enlaces considerados.

Para resolver o RMSA, para mais de um período de tráfego incremental, propomos o Algoritmo 1 que recebe, como entrada, uma matriz de tráfego $T=\left\{t_{i j} \in \mathbb{N}^{v \times v}\right\}$, onde cada elemento $t_{i j}$ representa a demanda incremental de transmissão, em bits por segundo, do nó $i$ para o nó $j$, onde $v$ é o número de nós na rede. $\mathrm{O}$ algoritmo também recebe uma estrutura $E$ contendo todos os enlaces $e_{i j}$ da rede e a estrutura $M$, contendo os FdMs que poderão ser usados. 


\subsection{Notações e Variáveis}

- $T$ : Matriz de demanda de tráfego $t_{i j}$ do nó $i$ para o nó $j . T=\left\{t_{i j} \in \mathbb{N}^{v \times v}\right\}$

- $E$ : Estrutura que contém todos os enlaces da rede, representados por $e_{i j}$, assim como as distâncias $\left|e_{i j}\right|$ e o número de slots de frequência ocupados $\left\|e_{i j}\right\|$, do enlace que conecta o nó $i$ ao nó $j$.

- $M$ : Estrutura que contém os formatos de modulação disponíveis $\eta_{m}$, suas respectivas distâncias máximas $\left|\eta_{m}\right|$ e seus respectivos valores de eficiência $\left\|\eta_{m}\right\|$. $M=\eta_{m} \cup\left\{\left|\eta_{m}\right| \in \mathbb{N}^{m}\right\} \cup\left\{\left\|\eta_{m}\right\| \in \mathbb{N}^{m}\right\}$

- $x$ : Valor númerico para contar o número de alocações bem sucedidas

- $p^{o}$ : Estrutura que contém o caminho escolhido pela heurística de RMSA, junto com sua distância total $\left|p^{o}\right|$ e sua carga máxima $\left\|p^{o}\right\|$

- $P_{i j}$ : Estrutura com os $k$ menores caminhos $p_{i j}^{k}$ entre nó $i$ e nó $j$.

- $p_{i j}^{k}$ : Estrutura que representa o $k$-ésimo menor caminho do nó $i$ para o nó $j$. Essa estrutura contém os enlaces $e_{i j}^{k}$ que fazem parte desta rota, a distância total $\left|p_{i j}^{k}\right|$ e a carga máxima $\left\|p_{i j}^{k}\right\|$ do $k$-ésimo caminho. $p_{i j}^{k}=\left\{e_{i j}^{k} \subset E\right\} \cup\left\{\left|p_{i j}^{k}\right| \in\right.$ $\left.\mathbb{N}^{k \times v \times v}\right\} \cup\left\{\left\|p_{i j}^{k}\right\| \in \mathbb{N}^{k \times v \times v}\right\}$

- $s$ : Variável booleana que assume o valor verdadeiro (true) ou falso (false), a partir do resultado da função $A$.

- $K$ : Função para calcular os $k$ menores caminhos do nó $i$ para o nó $j$, a partir da demanda $t_{i j}$ junto com a estrutura da rede, $E$.

- $A$ : Função que recebe uma demanda de tráfego $t_{i j}$, os elances $E$ e a estrutura que contém os FdMs disponíveis $M$ e tenta fazer o RMSA.

- $B$ : Valor numérico representativo da banda usada por cada SF.

- $G$ : Valor numérico da banda de guarda entre dois SFs alocados.

- F : Função de First Fit. Recebe o caminho escolhido $p^{o}$ e a carga do caminho escolhido $\tau^{o}$ e fará a alocação de SFs, de acordo com a política de alocação conhecida como First Fit.

- máx : Função que recebe vários valores e retorna o maior deles.

- mín : Função que recebe vários valores e retorna o menor deles.

\subsection{Algoritmo e Heurísticas}

O objetivo, do algoritmo é descobrir a taxa de bloqueio da rede, para cada período de incremento de tráfego. Para isso, precisamos do número de alocações bem sucedidas da rede, que é contado com a variável $x$, inicializada como zero (Linha 1). Então, para cada demanda de tráfego $t_{i j}$ dentro da matriz de tráfego $T$, usa-se uma função $A$, para fazer o RMSA (Linha 3). Caso a alocação seja possível, a função $A$ retornará um valor verdadeiro para a variável $s$ e a variável $x$ será incrementada de um (Linhas 3-6). O que queremos desse algoritmo é o número de alocações realizadas $x$ e os elances $E$ após feita a alocação. Para simular tráfego incremental com esse algortimo, basta usar o mesmo conjunto de enlaces $E$, os mesmos formatos de modulação $M$ e uma nova matriz de demanda de tráfego $T$.

O Algoritmo 1 usa a função $A$, que é uma heurística de resolução do RMSA. Aqui, apresentamos dois algoritmos heurísticos, com o objetivo de minimizar diferentes métricas na rede. O Algoritmo 2 é a primeira heurística e seu modo de operação é bastante simples. Esse algoritmo irá sempre escolher o caminho mais curto $p^{o}$, obtido usando o algoritmo de Dijkstra, que fornece os enlaces $e_{i j}$ que fazem parte do caminho mais curto 


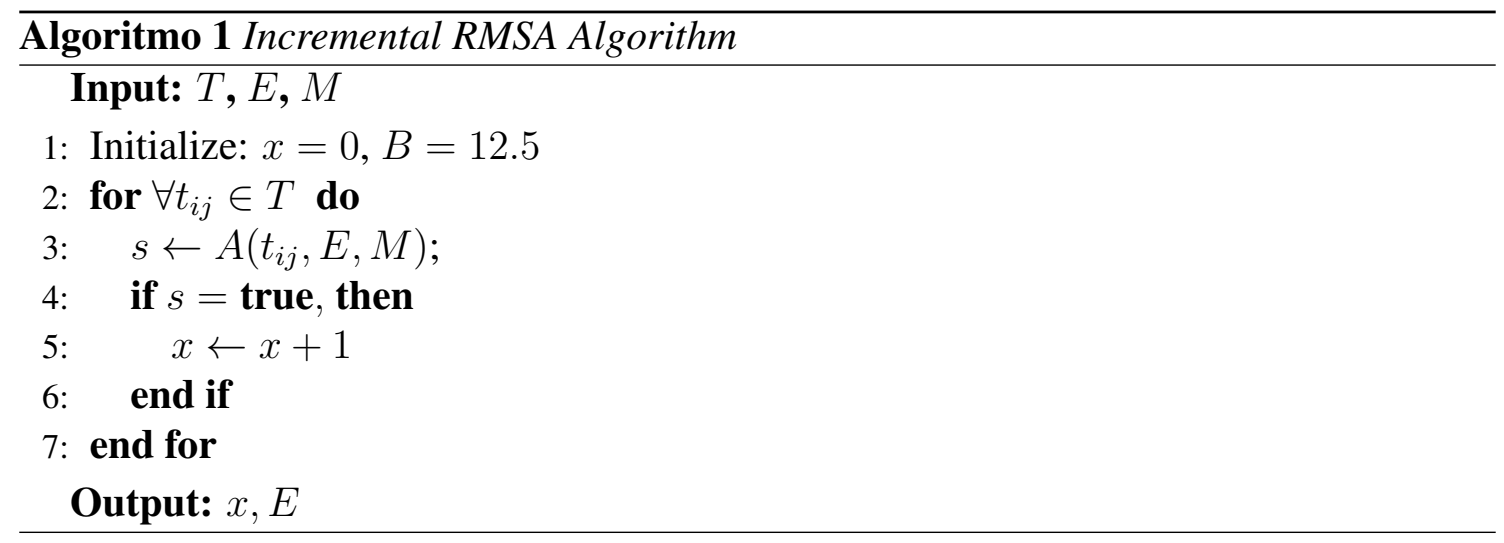

do nó $i$ ao no $j$ (Linha 2). A distância total $\left|p^{o}\right|$, do caminho escolhido, é tida como a somatória de todas as distâncias de cada um dos enlaces $e_{i j}$ que fazem parte de $p^{o}$ (Linha 3). A melhor eficiência $\left\|\eta^{o}\right\|$ é, então, escolhida, sendo a máxima das eficiências $\left\|\eta_{m}\right\|$ disponíveis, dado que a distância total $\left|p^{o}\right|$ do caminho escolhido é menor ou igual à distância máxima aceitável $\left|\eta_{m}\right|$ para este formato de modulação (Linha 4). Logo após, o tráfego, em número de slots, para o caminho escolhido será calculado com a Fórmula 1, usando a demanda, em bps, a eficiência do caminho escolhido e a banda usada por cada slot de frequência, $B$ e somando à banda de guarda $G$ (Linha 5). No final, o algoritmo clássico de alocação de SFs, First-fit (Por se tratar de um algoritmo conhecido, oculta-se a implementação), denominado de $F$ é aplicado (Linha 6). Assume-se que o algoritmo de First-fit retorne um valor verdadeiro, para $s$, caso a alocação seja bem sucedida e falso, caso contrário. O Algortimo 2 foi nomeado de Shortest Path with Minimum Load Modulation (SPMLM).

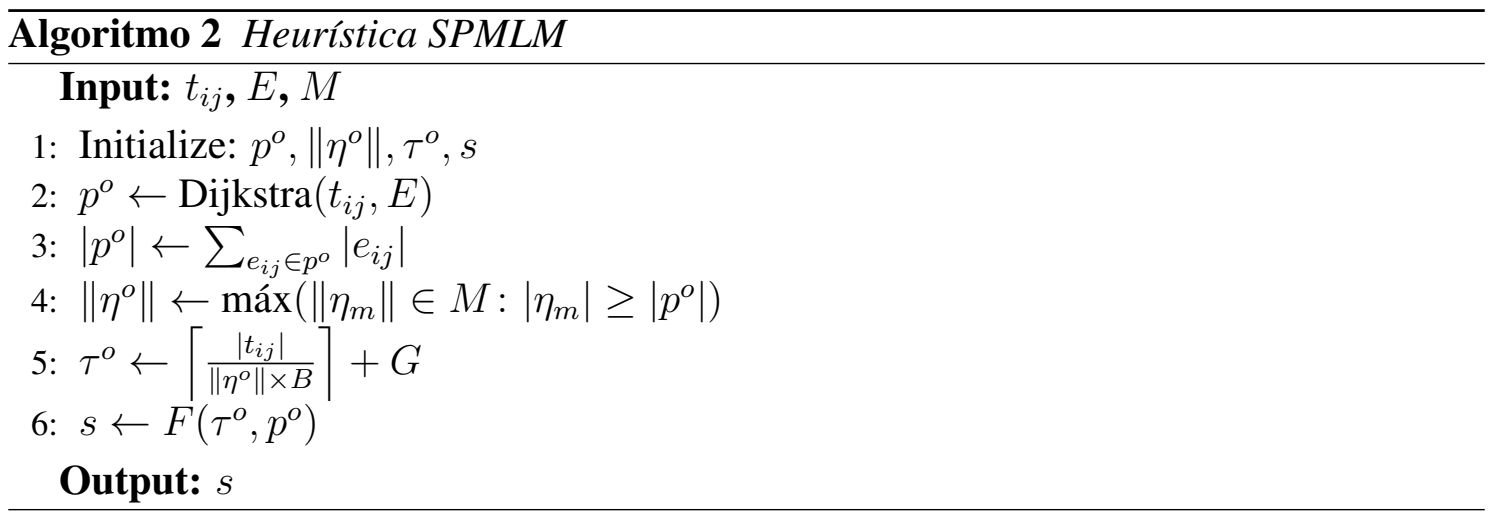

O outro algoritmo, apresentado neste trabalho, que pode ser usado como heurística da função $A$ é o Algoritmo 3, cujo objetivo é minimizar a carga máxima em qualquer um dos enlaces da rede. De início, se inicia as variáveis para guardar o caminho escolhido $p^{o}$, sua distância total $\left|p^{o}\right|$ e sua carga máxima $\left\|p^{o}\right\|$. Essas variáveis serão úteis mais à frente. Inicia-se também $\tau^{o}$ e $s$. Como esse algoritmo usa $k$ caminhos para uma determinada demanda, é necessário inicializar uma eficiência $\left\|\eta_{i j}^{k}\right\|$, assim como um tráfego, em número de slots $\tau_{i j}^{k}$, para cada caminho (Linha 1).

O segundo passo dessa heurística é calcular os $k$ menores caminhos possíveis para a demanda $t_{i j}$, através da função $K$ (Linha 2). A função $K$ pode ser implementada através de um algoritmo de $k$ menores caminhos, como aquele proposto em [Yen 1971]. Esses 
caminhos ficarão na estrutura $P_{i j}$. Logo após, para cada um dos caminhos $p_{i j}^{k}$, dentro de $P_{i j}$ (Linha 3), é calculada a distância total daquele caminho, como a somatória das distâncias individuais $\left|e_{i j}\right|$ de cada enlace pertencente a esse caminho (Linha 4). É calculado, também, a carga máxima deste caminho, como a carga máxima $\left\|e_{i j}\right\|$ dos enlaces que pertencem a este caminho (Linha 5). Em seguida, define-se, a melhor eficiência possível $\left\|\eta_{i j}^{k}\right\|$ dos FdMs possíveis, tal que a distância máxima do caminho seja menor ou igual à distância máxima aceita para aquele formato de modulação (Linha 6). Com a eficiência definida para cada caminho, calcula-se a carga que aquela demanda $t_{i j}$ irá exigir, caso seja roteada pelo caminho $p_{i j}^{k}$, com o uso da Fórmula 1, somada à banda de guarda $G$ (Linha 7).

Com $\left|p_{i j}^{k}\right|,\left\|p_{i j}^{k}\right\|,\left\|\eta_{i j}^{k}\right\|$ e $t_{i j}^{k}$ obtidos para cada $p_{i j}^{k}$. Segue-se, a carga máxima do caminho escolhido $\left\|p^{o}\right\|$ é tida como a carga do caminho no qual, a soma da carga máxima $\left\|p_{i j}^{k}\right\|$ com seu respectivo tráfego $t_{i j}^{k}$ é a menor entre todas (Linha 9). Escolhida a menor carga $\left\|p^{o}\right\|$, escolhe-se a menor distância total, entre as distâncias totais dos caminhos que possuem carga máxima igual a $\left\|p^{o}\right\|$ (Linha 10). Assim, o caminho mais curto, com menor carga máxima é o escolhido $p^{o}$ (Linha 11). O tráfego, em número de slots $\tau^{o}$ será o tráfego do caminho $p_{i j}^{k}$, ou seja, $\tau_{i j}^{k}$ (Linha 12). Então o algoritmo de First Fit é utilizado para alocar a carga neste caminho (Linha 6). O Algoritmo 3 foi chamado de Balanced Minimum Load Modulation (BMLM).

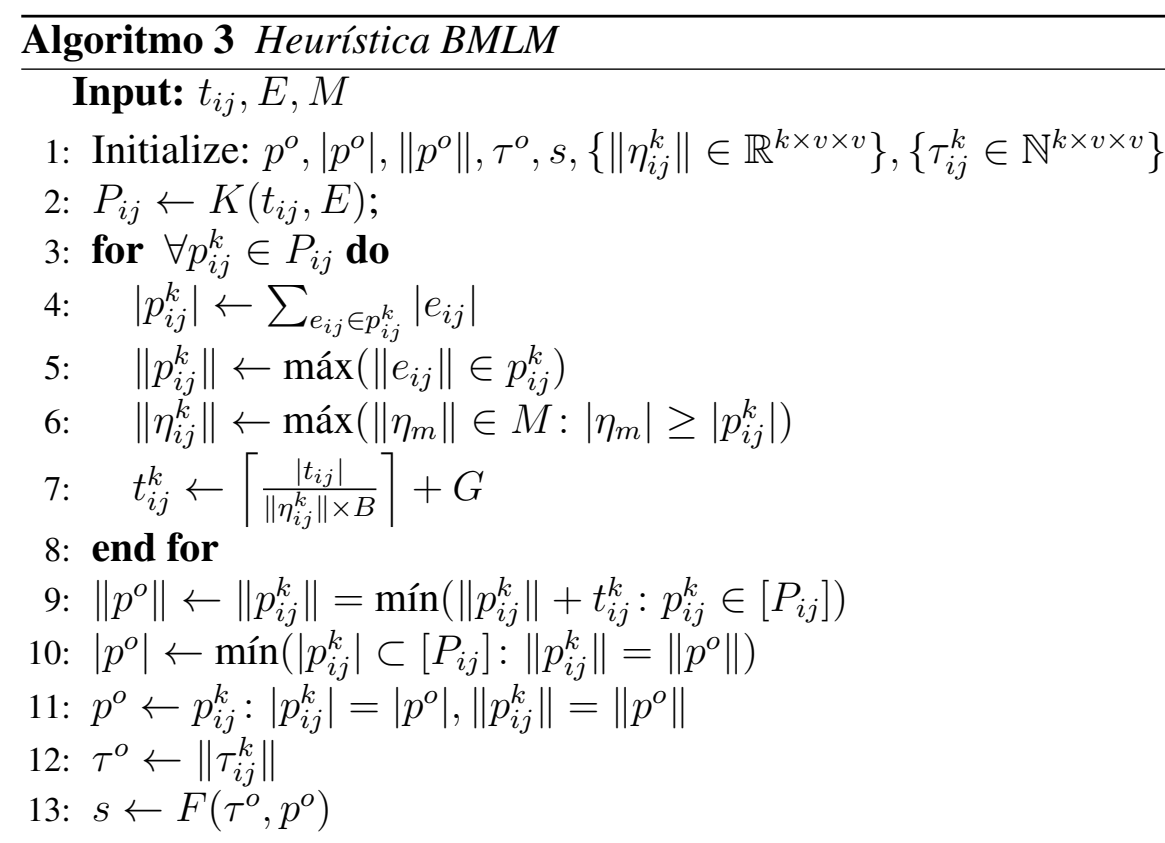

Output: $s$

\section{Simulações e Resultados}

O Algoritmo 1 da Seção 4 foi aplicado em 23 redes conhecidas, com diferentes números de nós e de enlaces. Foram simulados 25 períodos de tráfego incremental, alimentando 25 matrizes de tráfego $T$ ao algoritmo. Cada uma das matrizes de tráfego foi gerada como uma matriz de mesmas dimensões que a matriz de custo da rede, onde cada elemento $t_{i j}$ pode assumir valores entre 0, 100, 200, 300 e $400 \mathrm{Gbps}$, com uma distribuição uniforme de probabilidade. 
Para cada uma das redes, as matrizes de tráfego foram inseridas sequencialmente como entrada, para simular os períodos individuais. O número de conexões bem sucedidas $x$ foi usado para calcular a TdB relativo ao número total de conexões presentes em cada uma das matrizes de tráfego $T$.

Gerando a matriz $T$ desta forma, o tráfego total na rede será maior, quão maior for o número de nós da rede. Por isso, o número máximo de SFs em cada enlace foi diferente para redes de tamanhos diferentes. A quantidade máxima de SFs para cada rede e a lista das redes utilizadas podem ser encontrados na Tabela 2. Para uma mesma rede, o número máximo de SFs foi igual para todos os enlaces.

As redes foram modeladas a partir da sua matriz de custo, onde o custo $\left|e_{i j}\right|$ de cada enlace da rede é igual à sua distância. Dessa forma, o custo total de uma rota será a distância percorrida por todos os enlaces entre o nó $i$ e o nó $j$. Em termos de distância, para as simulações, foi considerado que cada enlace possui $500 \mathrm{~km}$.

Tabela 2. Redes e Parâmetros de Simulação

\begin{tabular}{ccccccc}
\hline \multicolumn{7}{c}{ Número de Nós na Rede (Primeira Parte) } \\
\hline 9 & 10 & 11 & 12 & 14 & 15 & 17 \\
\hline Via & BREN & Abilene & CESNET & Italiana & ACONET & Germany \\
\hline- & LEARN & CompuServe & VBNS & NFSNET & - & Spain \\
\hline- & RNP & - & - & - & - & - \\
\hline 512 & 512 & Numero Máximo de SFs por Enlace da Rede & \\
\hline & 512 & 512 & 512 & 512 & 1024
\end{tabular}

\begin{tabular}{ccccccc}
\hline \multicolumn{7}{c}{ Numéro de Nós na Rede (Segunda Parte) } \\
\hline 19 & 20 & 21 & 23 & 24 & 25 & 26 \\
\hline CANARIE & ARPANET & PIONIER & Bulgaria & COX & SANET & NewNet \\
\hline EON & Sweden & - & - & - & - & - \\
\hline Memorex & - & - & - & - & - & - \\
\hline \multicolumn{7}{c}{ Numero Máximo de SFs por Enlace da Rede } \\
\hline 1024 & 1024 & 2048 & 2048 & 2048 & 2048 & 2048
\end{tabular}

Primeiramente, as simulações foram realizadas usando o SPMLM e o BMLM, considerando apenas o formato de modulação de maior alcance, da Tabela 1, o BPSK, ou seja, $M=\left\{\left|\eta_{4}\right|=4000 \mathrm{~km},\left\|\eta_{4}\right\|=1\right\}$. Para ilustrar os resultados, foi escolhido o diagrama de caixas, por mostrar a tendência geral, desconsiderando outliers (Pontos fora da curva), para a ampla faixa de valores gerados nas simulações na gama de redes utilizadas. Os resultados são apresentados na Figura 2.

A Figura 2 mostra dois diagramas de caixas, lado a lado, um para os resultados do SPMLM e outro para o BMLM. Esse diagrama ilustra, para todas as redes, a faixa de valores de $\mathrm{TdB}$, assim como sua tendência, para cada período de incremento de tráfego. Às tendências são similares, mas é possível ver pela Figura 2 que a partir do segundo período, o BMLM, em geral, apresenta taxas de bloqueio maiores que o SPMLM, mas durante os 2 primeiros períodos, o BMLM apresentou taxas de bloqueio inferiores.

Este fenômeno ocorre por que o BMLM, ao tentar balancear as cargas, evita que alguns poucos enlaces fiquem sobrecarregados, em relação aos demais. O SPMLM, ao 


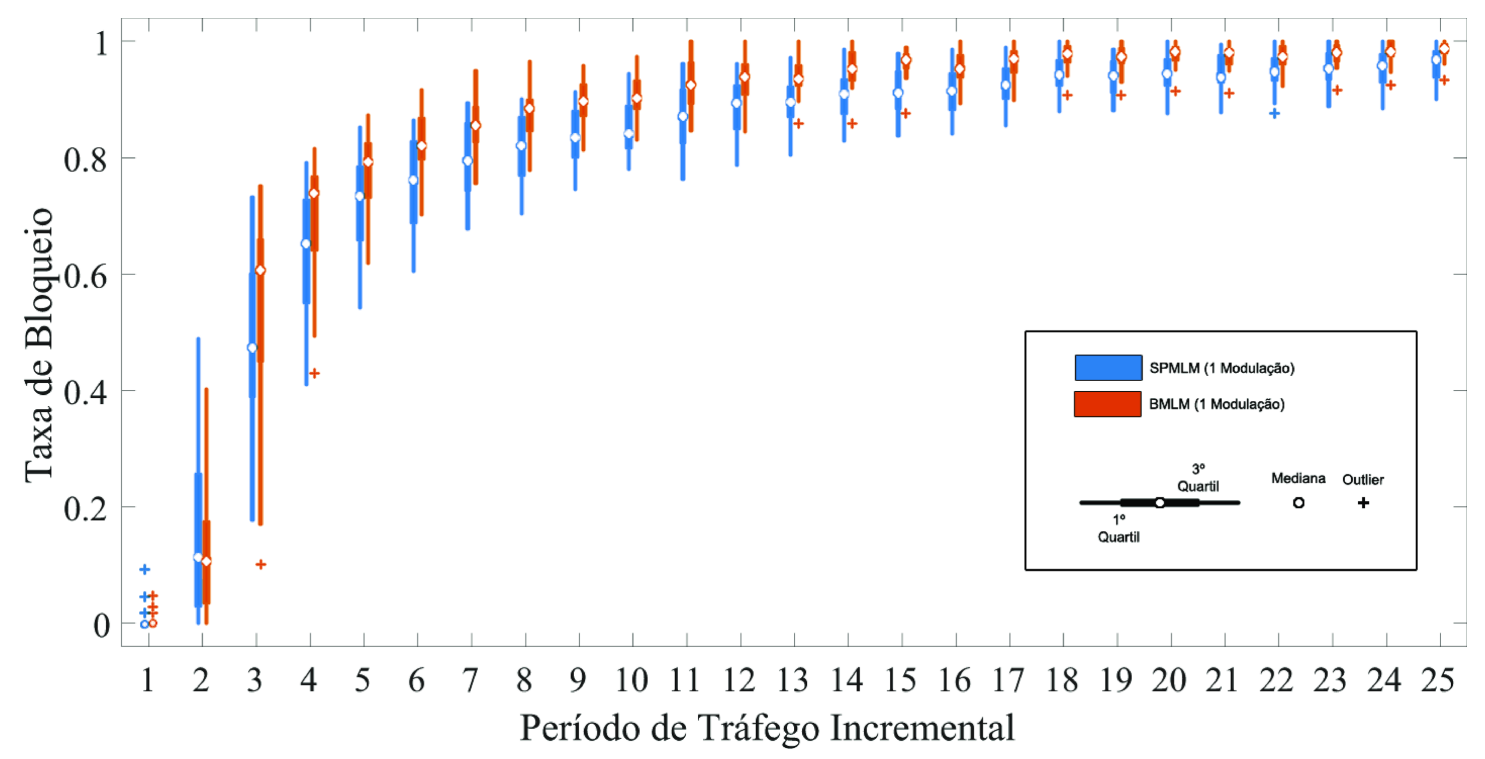

Figura 2. Gráfico de caixa para as taxas de bloqueio relativo a cada período de tráfego incremental simulado. Um formato de modulação disponível (BPSK). SPMLM e BMLM

sempre rotear as conexões pelo mesmo caminho, tem a tendência de usar alguns enlaces mais que outros e assim, tem uma maior probabilidade de bloquear mais tráfego no início. No entanto, na tentativa de balancear a carga, o BMLM usará caminhos mais longos, consumindo portadoras em um número maior de enlaces. Somado a esse fator, por usar rotas diferentes a todo o momento, as redes administradas pelo BMLM estarão sujeitas a maior fragmentação de espectro.

As simulações também foram feitas para as quatro opções de FdM, da Tabela 1, disponíveis. $M=\left\{\left|\eta_{1}\right|=500 \mathrm{~km},\left\|\eta_{1}\right\|=4,\left|\eta_{2}\right|=1000 \mathrm{~km},\left\|\eta_{2}\right\|=3,\left|\eta_{3}\right|=\right.$ $\left.2000 \mathrm{~km},\left\|\eta_{3}\right\|=2,\left|\eta_{4}\right|=4000 \mathrm{~km},\left\|\eta_{4}\right\|=1\right\}$. Os resultados estão na Figura 3.

Como esperado, ao comparar a performance das simulações com apenas um FdM disponível com as simulações com quatro FdMs, as taxas de bloqueio são menores para o último caso, mas também há maior disparidade dos valores, para os mesmos períodos, o que significa que algumas redes se beneficiaram mais que outras, por ter um número maior de opções de modulação.

É notavel que a performance do BMLM foi efetivamente pior que a do SPMLM sob essas condições de simulação. Isso ocorre porque, como os FdMs escolhidos pelos caminhos mais curtos são mais eficientes, existe um grande incentivo para rotas menores e o BMLM sofre maiores punições ao tentar balancear a carga por caminhos mais longos.

Apesar de apresentar pior performance geral, o BMLM obteve melhor taxa de bloqueio inicial em algumas poucas redes como a ACONET e a PIONIER, mostrando que a topologia pode influenciar nos resultados obtidos para um método de resolução do RMSA.

Para se ter uma visão mais completa de como as taxas de bloqueio se comportaram, em cada período, para o SPMLM e o BMLM, usando as duas diferentes conjunturas de FdMs, a Figura 4 possui todas as curvas das TdBs médias, para todas as simulações. 


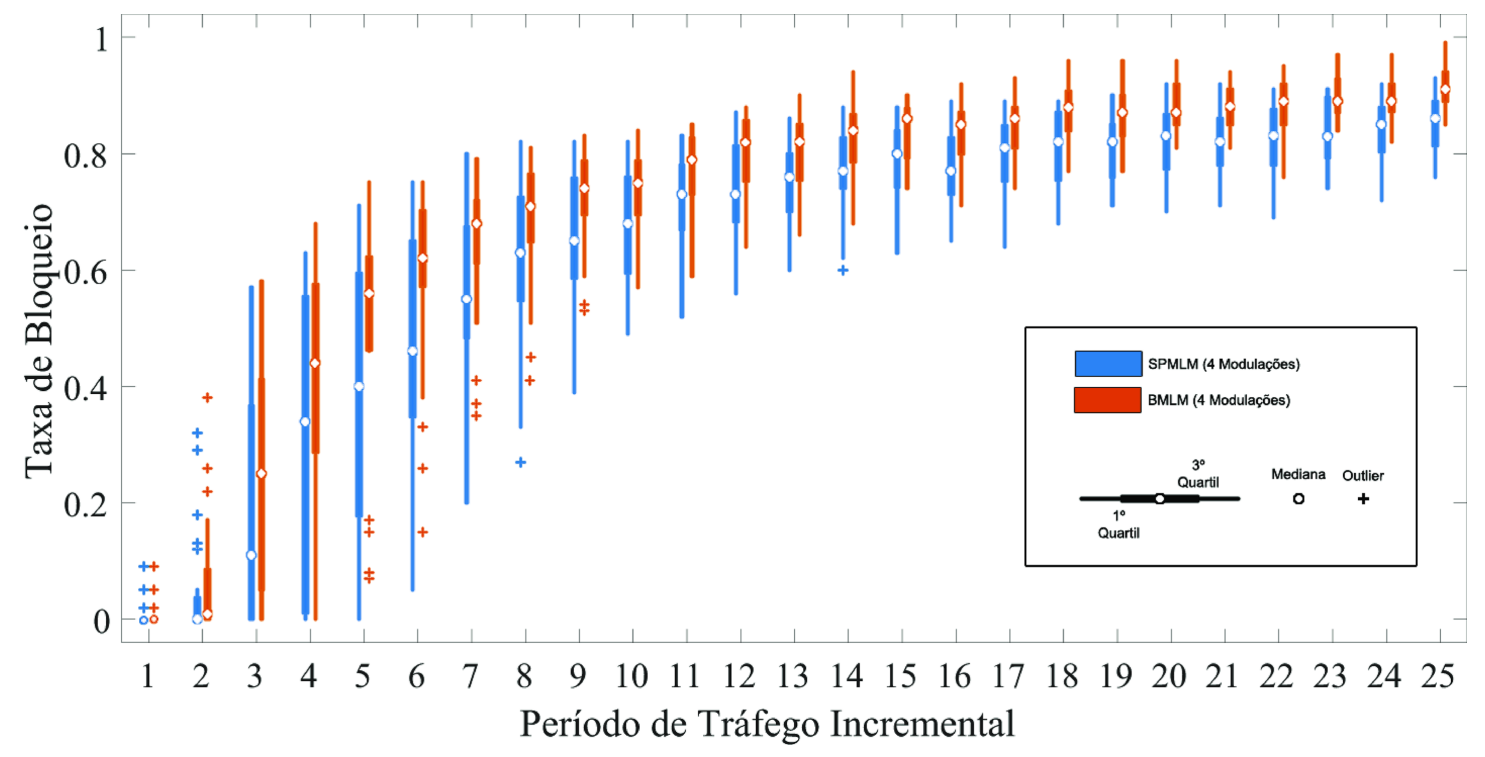

Figura 3. Gráfico de caixa para as taxas de bloqueio relativo a cada período de tráfego incremental simulado. Quatro formatos de modulação disponíveis. SPMLM e BMLM

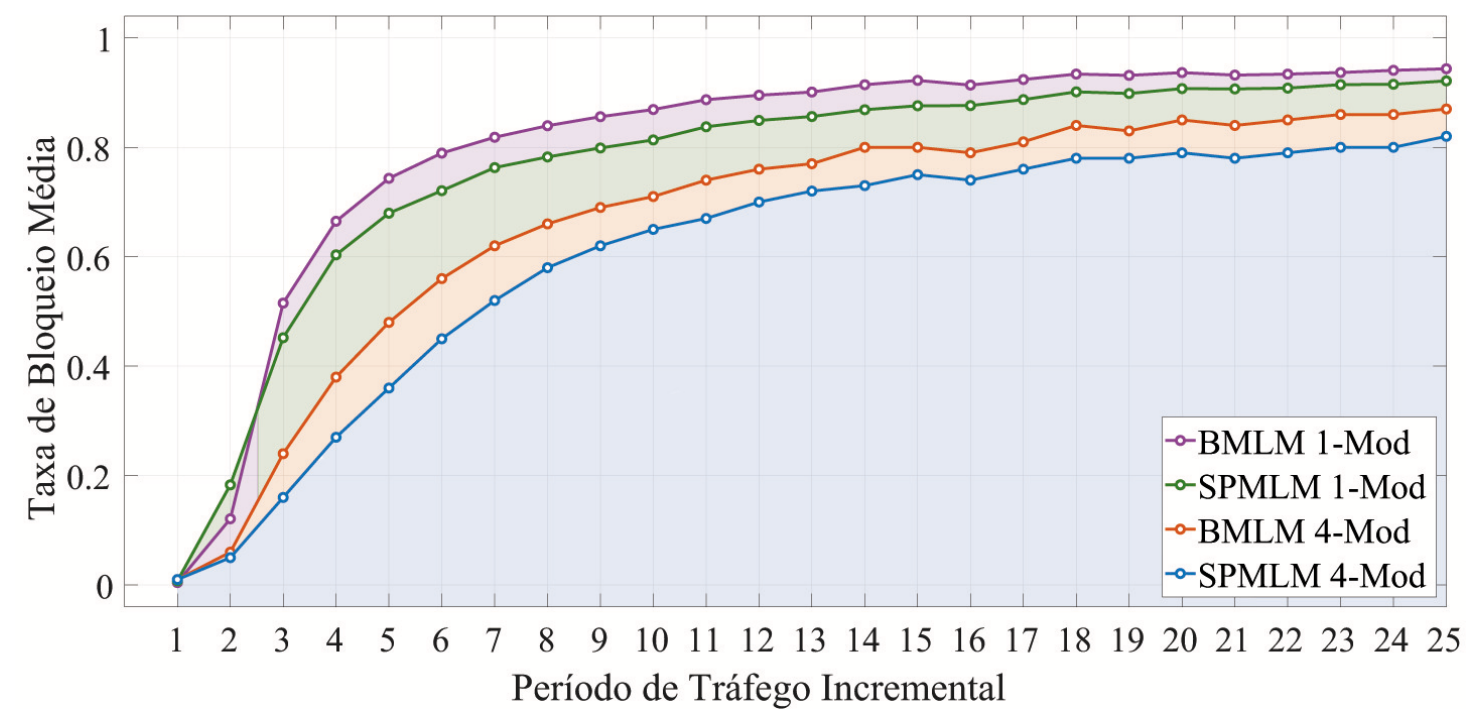

Figura 4. Curvas das taxas de bloqueio médias para cada período de tráfego incremental

\section{Conclusão}

Este artigo faz um estudo sobre o comportamento da taxa de bloqueio, em diversas redes, em simulações com tráfego incremental, para se obter dados sobre como o algoritmo de RMSA usado pode influenciar na performance de diferentes redes ao longo do tempo. Implementou-se duas heurísticas de RMSA. Uma de alocação por caminho mais curto (SPMLM) e uma heurística de balancemaneto de carga (BMLM), ambas as heurísticas foram comparadas em um cenário onde novas demandas de tráfego, estatisticamente previsíveis, chegavam em intervalos constantes de tempo.

Quando se considerou apenas um formato de modulação disponível, o BMLM obteve melhores taxas de bloqueio inicial, no entanto, sempre apresentou maiores taxas de 
bloqueio após esses primeiros períodos. Esse fenômeno é justificado pelo maior esgotamento da rede e maior fragmentação de espectro promovido pelo algoritmo. No entanto, é importante lembrar que uma menor taxa de bloqueio inicial pode ser útil para administradores de redes buscando adiar a manutenção de sua rede. Neste caso, seria recomendado usar o BMLM até que sua taxa de bloqueio fosse superior e então, substituir a heurística pelo SPMLM.

No cenário onde quatro formatos de modulação podiam ser usados pelas redes, não se pôde encontrar nenhuma vantagem em usar o BMLM, exceto em algumas poucas redes. A taxa de bloqueio média foi maior em todos os períodos de incremento de tráfego, pois a capacidade de caminhos mais curtos de usarem formatos de modulação com maior eficiência, tornou o SPMLM uma opção mais vantajosa. Mesmo nos períodos iniciais, o BMLM não foi capaz de trazer melhores taxas de bloqueio. No entanto, o BMLM conseguiu atrasar o início da taxa de bloqueio em duas das vinte e três redes, o que nos leva a frisar a importância de se fazer simulações dedicadas para qualquer rede específica que se deseje prever a performance, pois a rede pode se comportar de forma imprevisível em realação às tendências gerais, como apresentadas neste trabalho.

\section{Agradecimentos}

O presente trabalho foi realizado com apoio do Conselho Nacional de Desenvolvimento Científico e Tecnológico (CNPq) e da Coordenação de Aperfeiçoamento de Pessoal de Nível Superior - Brasil (CAPES) - Código de Financiamento 001. Agradecemos também ao suporte educacional da Universidade Federal da Bahia (UFBA).

\section{Referências}

Abkenar, F. S., and Rahbar, A. G. (2017). Study and analysis of routing and spectrum allocation (RSA) and routing, modulation and spectrum allocation (RMSA) algorithms in elastic optical networks, Opt. Switch. and Netw., vol. 23, pp. 5-39.

Agrawal, A., Vyas, U., Bhatia, V., and Prakash, S. (2017). SLA-aware differentiated QoS in elastic optical networks, Optical Fiber Technology, vol. 36, pp. 41-50, 2017.

Wang, Y., Cao, X., and Pan, Y. (2011). A Study of the Routing and Spectrum Allocation in Spectrum-Sliced Elastic Optical Path Networks. 2011 Proceedings IEEE INFOCOM.

Agrawal, G. (2013). Fiber-optic communication systems. Hoboken, N.J.: Wiley.

Cisco Systems. (2017). The Zettabyte Era: Trends and Analysis, available at https://www.cisco.com/c/en/us/solutions/collateral/service-provider/visual-networkingindex-vni/vni-hyperconnectivity-wp.pdf, June, 2017.

Christodoulopoulos, K., Tomkos, I., and Varvarigos., E. A. (2011). "Elastic bandwidth allocation in flexible ofdm-based optical networks," Journal of Lightwave Technology, vol. 29, no. 9, pp. 1354-1366.

Fontinele, A., Santos, I., Larcerda, J., Soares, A., Monteiro, J. S. (2018). Campelo, D. R., Alocação de Espectro com Redução de Interferências entre Circuitos em Redes Ópticas Elásticas. Anais do Simpósio Brasileiro de Redes de Computadores e Sistemas Distribuídos (SBRC), [S.1.], v. 36, may 2018. ISSN 2177-9384.

Telecommunication Standardization Section of ITU. (2002). Spectral grids for WDM applications: DWDM frequency grid, ITU-T G.694.2. 
Jaya, T., Gopinathan, E., and Rajendran, V. (2016). Comparison of BER Performance of Various Adaptive Modulation Schemes in OFDM Systems. Indian Journal of Science and Technology, vol. 9, no. 40.

Jia, X., Ning, F., Yin, S., Wang, D., Zhang, J., Huang, S. (2015). An Integrated ILP Model for Routing, Modulation Level and Spectrum Allocation in The Next Generation DCN. Third International Conference on Cyberspace Technology (CCT 2015).

Jinno, M., T, H., Kozicki, B., Tsukishima, Y., Sone, Y., and Matsuoka, S. (2009). Spectrum-Efficient and Scalable Elastic Optical Path Network: Architecture, Benefits, and Enabling Technologies, IEEE Communications Magazine, vol. 47, no. 11, pp. $66-73$.

Oliveira, Helder M. N. S., and Nelson L. S. Da Fonseca. (2017). Routing, Spectrum, Core and Modulation Level Assignment Algorithm for Protected SDM Optical Networks." GLOBECOM 2017 - 2017 IEEE Global Communications Conference.

Pioro, M., and Medhi, D. (2004). Routing, Flow, and Capacity Design in Communication and Computer Networks. Morgan Kaufmann.

Silva, K. A., Brasileiro, I. B., Costa, L. R., and Drummond, A. C. (2018). Estudo Sobre o Uso de Métricas de Fragmentação de Espectro no Projeto de Algoritmos RSA. Anais do Simpósio Brasileiro de Redes de Computadores e Sistemas Distribuídos (SBRC), [S.1.], v. 36, ISSN 2177-9384.

Straub, Stefan., Kirstadter, A., Schupke, D.A., "Multi-Period Planning of WDM-Networks: Comparison of Incremental and EoL Approaches." 2006 2nd IEEE/IFIP International Conference in Central Asia on Internet, 2006, doi:10.1109/canet.2006.279271.

Velasco, L., Morales, F., Gifre, L., Castro. A., Dios, G. O., and Ruiz, M., "On-demand incremental capacityplanning in optical transport networks, 'IEEE/OSA Jour-nal of Optical Communications and Networking, vol. 8,no. 1, pp. 11-22, January 2016.

Iyer, S. and Singh, P. S., "Multiple-Period Planning of Internet Protocol-over-Elastic Optical Networks." Journal of Information and Telecommunication, vol. 3, no. 1, 2018, pp. 39-56., doi:10.1080/24751839.2018.1526448.

Soumplis, P., Christodoulopoulos, K., Quagliotti, M., Pagano, A., Varvarigos, M. MultiPeriod Planning With Actual Physical and Traffic Conditions. Journal of Optical Communications and Networking, vol. 10, no. 1, 2017, doi:10.1364/jocn.10.00a144. 2017.

Yen, J. (1971). Finding the k shortest loopless paths in a network,'Management Science, vol. 17 , no. 11, pp. 712-16.

Zhang, G., Leenheer, M. D., Morea, A., and Mukherjee, B. (2013). A survey on ofdmbased elastic core optical networking, Commun. Surv. Tutorials, IEEE 15 (1) 65-87. 